\title{
A Probabilistic Approach to Susceptibility Measurement in a Reverberation Chamber
}

\author{
Emmanuel Amador, Christophe Lemoine, Philippe Besnier \\ IETR, UMR CNRS 6164, INSA Rennes, France \\ emmanuel amadordinsa-rennes. fr
}

\begin{abstract}
In this article, we propose an alternative approach for measuring the susceptibility level of an equipment under test in a reverberation chamber. Instead of using a statistical estimation of the maximum, our estimation is based on an estimation of the probability of failure of the equipment under test. We show through Monte Carlo simulations that the probability of failure of an equipment under test allows to predict with a good accuracy its susceptibility level. An experimental validation with a simple equipment under test shows that the susceptibility levels measured in a reverberation chamber are consistent with measurements performed in a GTEM cell.
\end{abstract}

\section{INTRODUCTION}

Susceptibility measurements in a reverberation chamber (RC) are based on a statistical estimation of the maximum level an equipment under test (EUT) may have received over a number $N$ of independent stirrer positions [1]. A lot of work [2], [3], [4], [5], [6], [7] has been done in the community to characterize statistically the estimation of the maximum value of a rectangular E-field component or the maximum power in an RC. Extreme value theory is used to determine the probability density function of the maximum power [2] or the maximum of a rectangular component of the E-field[4] in the chamber.

Let $X$ a random variable with a density probability function $f_{X}(x)$ and a cumulative distribution function $F_{X}(x)$. The cumulative distribution function of the maximum value over $N$ independent samples $F_{N}$ is given by:

$$
F_{N}(x)=F_{X}(x)^{N}
$$

and thus the density probability function of the maximum is given by:

$$
f_{N}(x)=N F_{X}(x)^{N-1} f_{X}(x) .
$$

The maximum value of the power or the maximum value of a rectangular component of the E-field is a $N$-order statistics. It is derived from the size $N$ of the sample measured (e.g the number of independent stirrer positions or the number of independent frequency used during the measurement) and the power injected in the RC. It means that by using an estimation of the maximum, an intrinsic quantity like the susceptibility level of an EUT is a function of the number of stirrer positions used during the test. Moreover, the uncertainty of the estimation of the maximum of a rectangular E-field component is large and decreases slowly with the size of the sample $N$ as shown in continuous line in figure 1. In this article we propose a new approach based on the probability

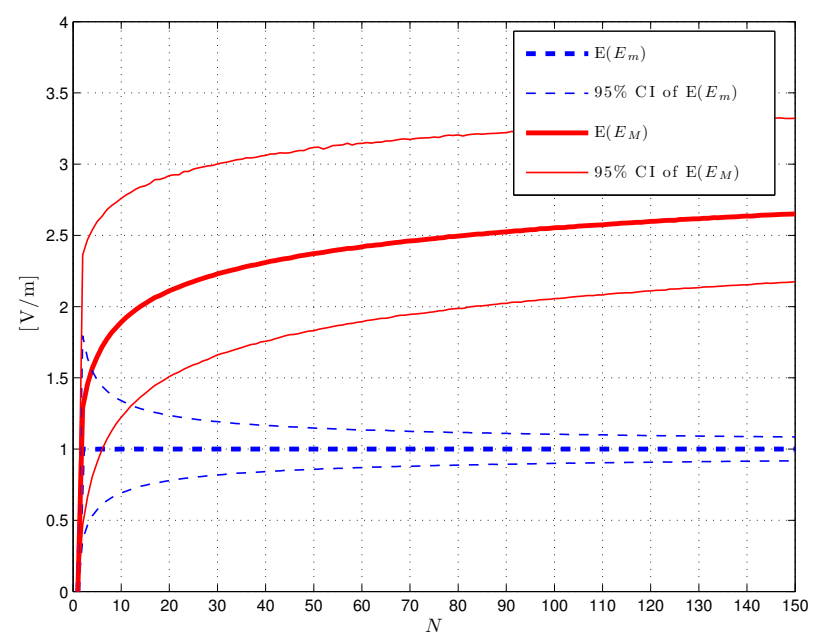

Fig. 1. Monte Carlo simulations ( $10^{5}$ experiments), showing the expected value and the $95 \%$ confidence interval $(\mathrm{CI})$ of both the mean value of the magnitude of a rectangular E-field component $E_{m}$ and the maximum magnitude of rectangular E-field component $E_{M}$ for different values of $N$ The mean value of the rectangular component is set to $1 \mathrm{~V} \cdot \mathrm{m}^{-1}$ in these simulations.

of failure of the EUT. This approach uses an estimation of the mean value of a component of the E-field during the measurement and an estimation of the probability of failure of the EUT during the testing. As shown in blue in figure 1, the uncertainty of an estimation of the mean value of a rectangular E-field component decreases more rapidly. After presenting the theoretical background of our approach, we show that it can be used to measure with a good accuracy the susceptibility level of an EUT. The approach is confirmed by an experimental validation and the values obtained are consistent with measurements performed in a guided wave setup like a GTEM cell.

\section{MEASUREMENT OF THE SUSCEPTIBILITY BASED ON THE PROBABILITY OF FAILURE OF AN EUT}

In this section, we propose a method that allows to derive the susceptibility of the EUT from the probability of failure measured in an RC. The statistical distribution followed by a rectangular E-field component in an overmoded and wellstirred RC is a Rayleigh distribution. The mean value of a Rayleigh distribution is $\sigma \sqrt{\frac{\pi}{2}}$ [8]. The cumulative probability function of a Rayleigh distribution with scale parameter $\sigma$ 


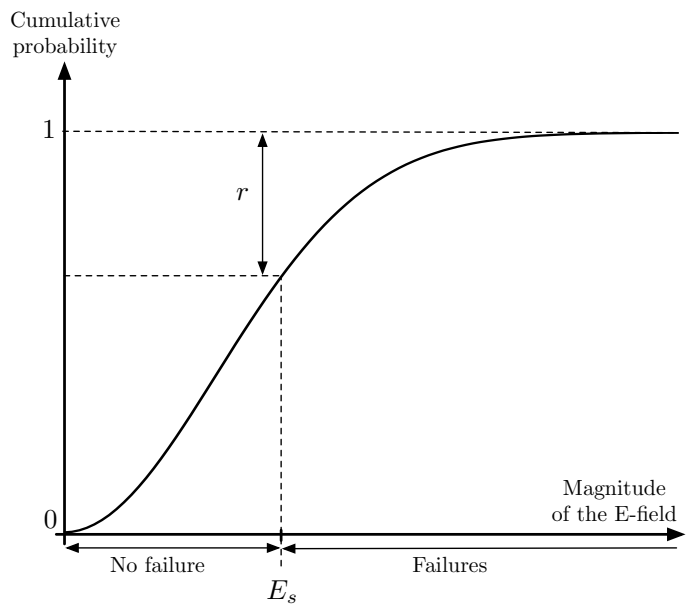

Fig. 2. Cumulative density function of a Rayleigh distribution. Above the susceptibility level $E_{s}$, failures are detected with a probability $r$.

(shown in figure 2) is given by [8]:

$$
F(x)=1-e^{-x^{2} / 2 \sigma^{2}} \text {, with } x \geq 0 .
$$

When the mean value of the E-field $E_{m}$ in the chamber equals the susceptibility level $E_{s}$ of the EUT, the probability of failure $r_{m}$ can be derived by posing:

$$
r_{m}=1-F\left(E_{s}\right)=1-F\left(\sigma \sqrt{\frac{\pi}{2}}\right)=e^{-\pi / 4} \approx 46 \%
$$

If a default is detected for $r_{m}=46 \%$ of the stirrer positions, the susceptibility level $E_{s}$ of the EUT is directly given by the mean value $E_{m}$ of a rectangular component of the E-field in the chamber.

Let define the susceptibility level $E_{s}$ of an object in an $\mathrm{RC}$ as the mean value of a rectangular component for which $e^{-\pi / 4} \approx 46 \%$ of the stirrer positions provoke a failure on the EUT.

We will derive a general relation that allows to deduce the susceptibility level $E_{s}$ from a measurement with an arbitrary mean value of a rectangular component of the E-field $E_{m}$ and a corresponding probability of failure of the EUT $r$. If $r>0.46$, the mean value of a rectangular E-field component $E_{m}$ is greater than the susceptibility level $E_{s}$ of the EUT, and if $r<0.46$, we can deduce that $E_{m}<E_{s}$. The knowledge of the probability of failure $r$, the mean value of the E-field $E_{m}$ in the chamber, and the theoretical CDF of an E-field component allow to predict the susceptibility level $E_{s}$. From (4), we can write:

$$
r=1-F\left(E_{s}\right)=e^{-E_{s}^{2} / 2 \sigma^{2}}
$$

We can write the two following equations:

$$
\left\{\begin{array}{r}
E_{s}=\sigma \sqrt{2 \ln (1 / r)} \\
E_{m}=\sigma \sqrt{\pi / 2} .
\end{array}\right.
$$

and thus:

$$
E_{s}=2 E_{m} \sqrt{\frac{\ln (1 / r)}{\pi}}
$$

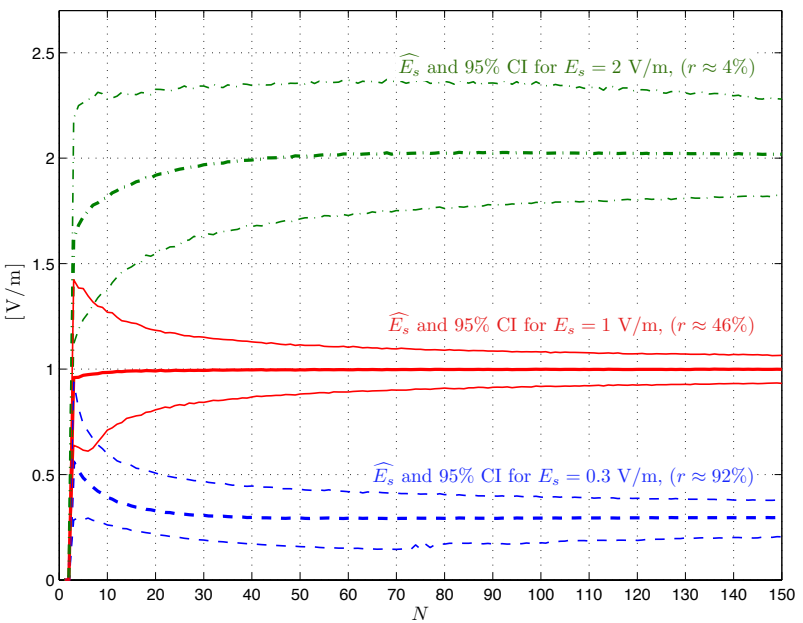

Fig. 3. Mean value and $95 \% \mathrm{CI}$ of the estimator $\widehat{E_{s}}$ for different values of susceptibility $E_{s}$ and for different values of $N$. The mean value of a rectangular component $E_{m}$ is set to $1{\mathrm{~V} . \mathrm{m}^{-1}}^{-1}$. Monte Carlo simulations with $10^{4}$ experiments for each value of $N$.

We can extract the level of susceptibility of an EUT from its probability of failure, roughly given by $\widehat{r}=N_{f} / N$ and from an estimation of the mean value the magnitude of a rectangular component of the electric field during the measurement $\widehat{E_{m}} 1$.

\section{MONTE CARLO SIMULATIONS OF OUR APPROACH}

In this section we study the statistical properties of our approach by using Monte Carlo simulations. The goal is to have an indication of the quality of the estimator $\widehat{E_{s}}$ of the level of susceptibility $E_{s}$. From equation (7), we can note that the estimator $\widehat{E_{s}}$ is a function of two estimators, $\widehat{E_{m}}$ and $\widehat{r}$. As these two estimators are estimated by computing an average over a sample of size $N$, the confidence interval (CI) should decrease with the sample size $N$.

\section{A. Effect of the sample size $N$ on the confidence interval}

Figure 3 shows the estimation of the susceptibility level for three different levels of susceptibility $E_{s}$ as a function of the number of stirrer positions $N$. In these simulations, the mean value of the E-field is set to $1 \mathrm{~V} \cdot \mathrm{m}^{-1}$. First in red, the susceptibility level equals the mean value of the Efield. As pointed out in (4), $46 \%$ of the stirrer positions provoke a failure on the EUT. The statistics of $\widehat{E_{s}}$ is similar to the statistics of $E_{m}$ presented in figure 1. We can note that the uncertainty of the estimation varies with the level of susceptibility measured. This is mostly due to the estimation of the probability of failure $r$. If $E_{s}=0.3 E_{m}$, as depicted in blue in figure 3 , the probability of failure equals $92 \%$. The CI remains large when the number of stirrer positions increases and the mean value of $\widehat{E_{s}}$ converges to $E_{s}$ when $N>20$. If $E_{s}=2 E_{m}$, as depicted in green in figure 3 , the probability of failure equals $4 \%$. The CI remains significant when the

${ }^{1}$ If the quality factor of the chamber with the EUT is known, $E_{m}$ can be derived directly from it. 


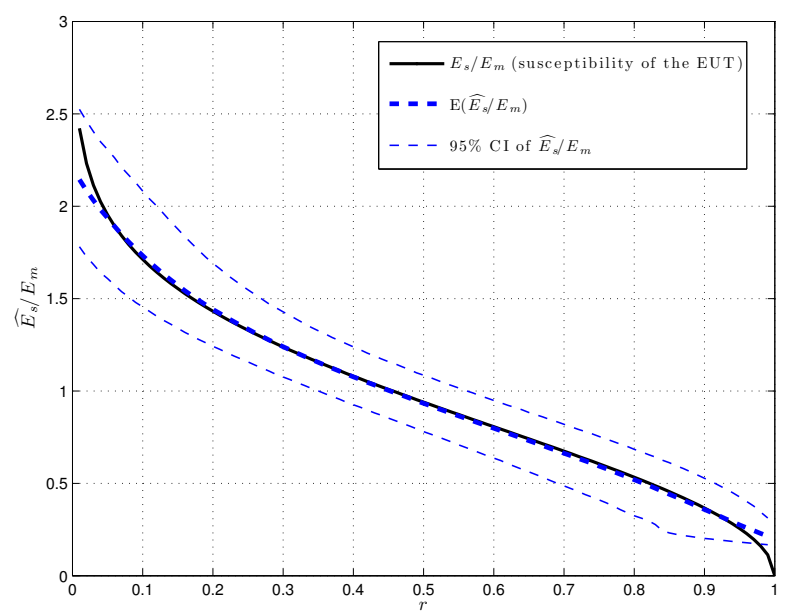

Fig. 4. Mean value and $95 \% \mathrm{CI}$ of the estimator $\widehat{E_{s}}$ normalized by $E_{m}$ as a function of the probability of failure $r$ with $N=30$ stirrer positions Monte Carlo simulations with $10^{5}$ experiments for each value of $r$.

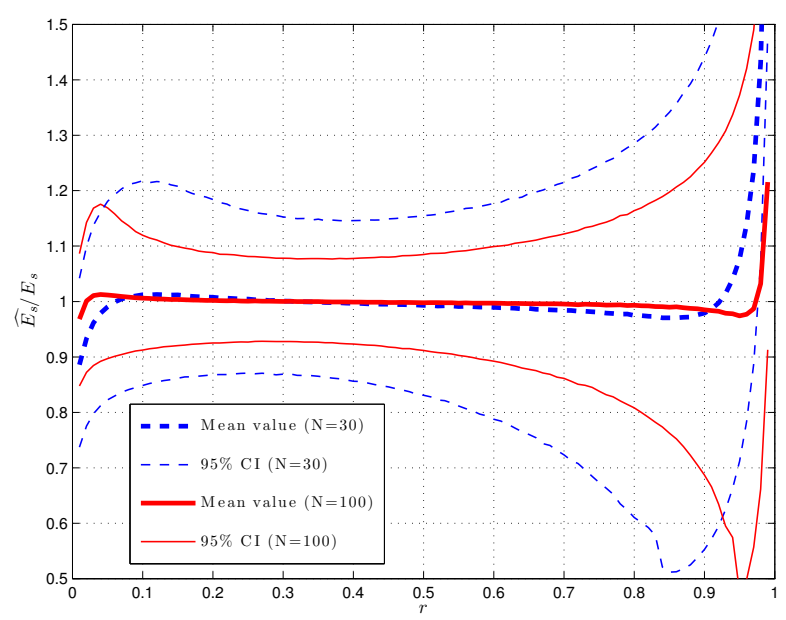

Fig. 5. Mean value and $95 \%$ CI of $\widehat{E_{s}} / E_{s}$ as a function of the probability of failure $r$ with $N=30$ and $N=100$ stirrer positions. Monte Carlo simulations with $10^{5}$ experiments for each value of $r$.

number of stirrer positions increases and the mean value of $\widehat{E_{s}}$ converges to $E_{s}$ if $N>40$.

These Monte Carlo simulations show that a probabilistic measurement of the susceptibility gives good results as long as the values of $r$ or $1-r$ are not too small. Unlike an estimation based on the maximum value of the E-field, this estimation based on its mean value does not increase with $N$.

\section{B. Effect of the probability of failure $r$ on the confidence interval}

Figure 4 shows the mean value and the $95 \% \mathrm{CI}$ of $\widehat{E_{s}} / E_{m}$ when $N=30$. We can note that with $N=30$ the mean value of the estimator is correct for a probability of failure $r$ between 0.05 and 0.95 . For a correct estimation of the susceptiblity level, one can use the following rule of thumb $\frac{2}{N} \leq r \leq 1-\frac{2}{N}$. Figure 4 shows that the absolute width of the CI interval of $\widehat{E_{s}} / E_{m}$ is not varying with $r$. It means that relative error

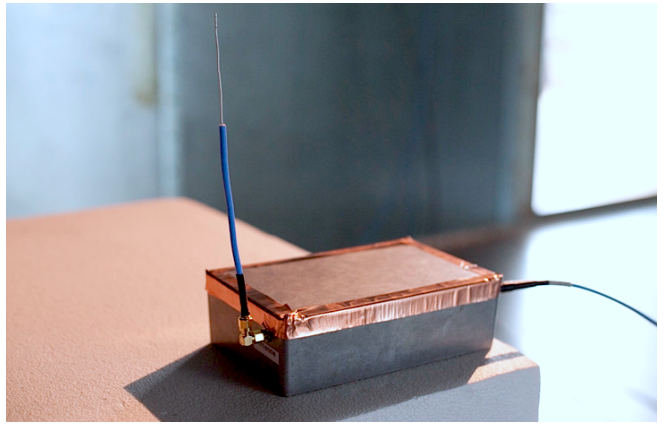

Fig. 6. External view of the equipment under test.

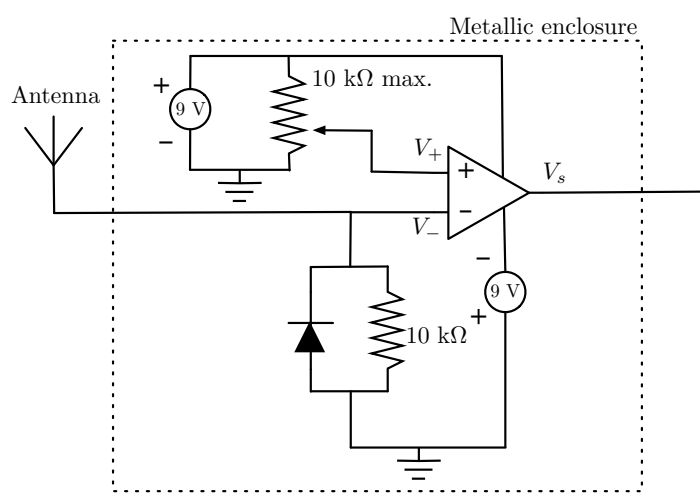

Fig. 7. Schematic view of the equipment under test and its electronic board.

is increasing when the susceptibility level $E_{s}$ is decreasing. Figure 5 shows the CI of the relative estimator $\widehat{E_{s}} / E_{s}$ for $N=30$ stirrer positions and $N=100$. If $N=30$, the relative error of $95 \%$ of the Monte Carlo experiments does not exceed $\pm 20 \%$ as long as $r \leq 0.6$. If $N=100, r$ should not exceed 0.8 .

These Monte Carlo simulations show that the estimation of a susceptibility level based on the probability of failure of an EUT is possible and may be a good alternative to measurements based on the maximum value. The uncertainty of the estimation is relatively well controlled and decreases more rapidly when $N$ is increasing than the estimation based on the maximum.

\section{EXPERIMENTAL VALIDATION}

\section{A. Experimental setup}

In order to validate our approach, we designed an EUT. This EUT consists of an electronic board with an operational amplifier (op-amp) acting as a comparator. The board is placed in a metallic enclosure. A $5 \mathrm{~cm}$ long monopole external antenna is connected to the circuit as shown in figure 6. A schematic of the electronic circuit is given in figure 7. The antenna is associated with an envelope detector for filtering the high frequency and for rectifying the signal. Without any disturbance, since $V_{+}>V_{-}$the op-amp delivers $V_{s}=9 \mathrm{~V}$. With disturbances leading to $V_{-}>V_{+}$, the op-amp provides $V_{s}=-9 \mathrm{~V}$ indicating a default. The signal $V_{s}$ is recorded with a digital oscilloscope and a home made program that 


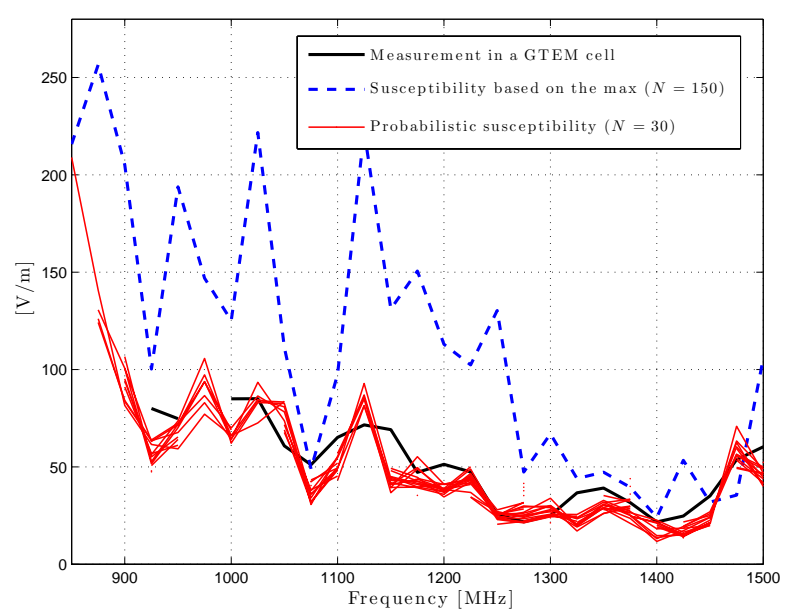

Fig. 8. Measurements of the susceptibility in an RC with our probabilistic approach for various injected power (or $E_{m}$ ) as calculated from (7) (in red), with the method based on an estimation of the maximum value of the E-field (in blue) and measurements of the susceptibility performed in a GTEM cell (in black).

controls all the experimental setup. The program returns either the value 0 in the case of no susceptibility, or the value 1 if a susceptibility is detected. The measurements are performed between $850 \mathrm{MHz}$ and $1500 \mathrm{MHz}$. At these frequencies, the behavior of our chamber is ideal and measurements have shown that the rectangular E-field components follow a Rayleigh distribution. We choose to use $N=150$ stirrer positions and the power injected in the chamber is increased

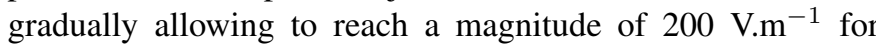
the rectangular components of the E-field. Susceptibility measurements were performed with the same setup in a GTEM cell at different positions in the test volume. Since the levels obtained from one position to another were similar, we choose to keep only the minimum value of the susceptibility among the different positions.

\section{B. Results}

Figure 8 shows the different susceptibility levels measured in the RC and in the GTEM cell of our laboratory. Our setup did not allow to get an E-field magnitude greater than $100 \mathrm{~V} \cdot \mathrm{m}^{-1}$ in the GTEM cell. It explains the lack of susceptibility detection below $1 \mathrm{GHz}$. The measurements of the susceptibility based on an estimation of the maximum magnitude of the E-field in the reverberation chamber with $N=150$ stirrer positions are given by the blue curve. In comparison with the measurements made in the GTEM cell, the susceptibility measured is generally higher and for some frequencies, the levels measured are superior by a factor of 4 . This susceptibility measurement exhibits oscillations that cannot be explained physically given the length of the antenna. The oscillations are mainly due to the statistical uncertainty in the estimation of the maximum value as explained in section I and presented in figure 1 . The red curves in figure 8 show the susceptibility of the EUT by using our approach with only $N=30$ stirrer positions. These curves correspond to the different levels of power injected in the chamber. We estimate the susceptibility if and only if the estimated probability of failure $\widehat{r}$ is between $2 / N$ and $1-2 / N$. These curves show that our probabilistic approach of the susceptibility levels give results that are consistent with the levels measured in the GTEM cell. If the EUT is not prone to be damaged easily, this approach can reduce the duration of the testing by determining almost immediately the susceptibility level of the EUT instead of increasing gradually the level of power injected in the chamber.

\section{Conclusion}

This article gives an alternative approach for measuring the susceptibility in an RC. This approach based on an estimation of the probability of failure $r$ of an EUT allows to predict with accuracy the level of susceptibility of an EUT. We based our investigation on a rectangular component of the electric field in an ideal reverberation chamber. Thus we used a Rayleigh distribution to build our approach. The transposition of this method with other distributions is straightforward, we can use Weibull distributions to perform susceptibility measurements at lower frequencies or exponential distributions if the quantity measured is the power received. The various Monte Carlo simulations show that the CI of this method decreases with the number of stirrer positions. The measurements on an EUT show that the susceptibility levels obtained with this probabilistic approach are consistent with measurements made in a GTEM cell and that the statistical dispersion of the values is reduced in comparison with measurements based on a statistical estimation of the maximum.

\section{ACKNOWLEDGMENT}

This work was supported by the French Ministry of Defence DGA (Direction Générale de l'Armement), with a Ph.D. grant delivered to Emmanuel Amador.

\section{REFERENCES}

[1] Reverberation Chamber Test Methods. International Electrotechnical Commission (IEC) Standard 61000-4-21, 2003

[2] T. Lehman and G. Freyer, "Characterization of the maximum test level in a reverberation chamber," in Electromagnetic Compatibility, 1997. IEEE 1997 International Symposium on, Aug. 1997, pp. 44 - 47.

[3] G. Koepke and J. Ladbury, "New electric field expressions for EMC testing in a reverberation chamber," in Digital Avionics Systems Conference, 1998. Proceedings., 17th DASC. The AIAA/IEEE/SAE, vol. 1, 1998, pp. D53/1 -D53/6.

[4] K. Harima, "Statistical characteristics of maximum E-field distribution in a reverberation chamber," in Electromagnetic Compatibility, 2004. EMC 2004. 2004 International Symposium on, vol. 2, 2004, pp. $724-727$.

[5] M. Hoijer, "Maximum power available to stress onto the critical component in the equipment under test when performing a radiated susceptibility test in the reverberation chamber," Electromagnetic Compatibility, IEEE Transactions on, vol. 48, no. 2, pp. 372 - 384, May 2006.

[6] M. Hoijer, H. Krauthäuser, and J. Ladbury, "On "maximum power available to stress onto the critical component in the equipment under test when performing a radiated susceptibility test in the reverberation chamber"," Electromagnetic Compatibility, IEEE Transactions on, vol. 50, no. 4 , p. 1020 , nov. 2008

[7] G. Orjubin, "Maximum field inside a reverberation chamber modeled by the generalized extreme value distribution," Electromagnetic Compatibility, IEEE Transactions on, vol. 49, no. 1, pp. 104 - 113, 2007.

[8] A. Papoulis, Probability, Random Variables, and Stochastic Processes, 4th ed. New York: Mc Graw Hill, 2002. 